\title{
Features, predictors and Management of Ostial Coronary Artery Diseases
}

\author{
O.S.Arafa, M.E.El Seteha, E.S.El Keshk, A.E.El Nagar and M.M.El Sawaf
}

Cardiology Dept., Faculty of Medicine, Benha Univ., Benha, Egypt

E-Mail: drsawaf445@gmail.com

\begin{abstract}
Ostial lesions have been recognized as a challenge from multiple perspectives for percutaneous coronary intervention [PCI]. Right off the bat, ostial injuries are well on the way to be related with problematic angiographic results because of injury inflexibility and force. Also, careful stent situating is a principle issue in performing PCI. Thirdly, troubles in the arrangement of a managing catheter and poor back-up help are different elements that may influence the conclusive outcome. To decide the highlights, indicators and the board of ostial coronary conduit ailments. The current examination assessed 2000 back to back patients alluded to the cardiology division of Benha college medical clinics and Tanta college Hospitals with ischemic chest torment experienced indicative catheterization. From those patients just 200 coordinated our populace and determined to have coronary ostial injury and experienced PCI. All patients had history taking, total physical assessment, ECG, Echo, research facility examinations and coronary angiography. As to the sorts of bifurcation strategies done in PCI, Culotte strategy was done in 28 patients, DK Crush procedure was done in 36 patients. Scaled down - Crush procedure was done in 52 patients, TAP strategy was done in 36 patients, V Stenting method was done in 16 patients lastly, Szabo strategy was done in 28 patients. Best outcomes and less difficulties were with Szabo procedure. Best outcomes and less inconveniences to oversee ostial injury were related with [Szabo] strategy, while more entanglements were related with [Culotte] procedure.
\end{abstract}

Key words: Coronary ostial lesion, Bifurcation lesion, PCI, Szabo technique.

\section{Introduction}

Percutaneous coronary intercession [PCI] in ostial injuries speak to a test to the interventional cardiologist since they frequently include the mass of the aorta, they are regularly calcified, they may not completely expand and they are inclined to restenosis. An ostial sore is characterized as an injury which starts inside 3-5 mm of the inception of a significant epicardial supply route [1]. It might be arranged by area as aorto-ostial, non aorto-ostial, or branch-ostial [2].

Ostial sores have been perceived as a test from various viewpoints for percutaneous coronary mediation [PCI]. Initially, ostial injuries are well on the way to be related with problematic angiographic results because of injury unbending nature and force. Besides, careful stent situating is a principle issue in performing PCI. Thirdly, troubles in the arrangement of a controlling catheter and poor back-up help are different variables that may influence the conclusive outcome [3] .

There are numerous procedures used to help in exact ostial stent arrangement; these incorporate utilizing various angiographic perspectives to aid situation, the utilization of the Ostial Pro gadget, the aorta streaming wire strategy, Szabo [anchor-wire] strategies, the T-stent and little distension [TAP] method, the traverse 1-stent strategy, and new committed ostial stents [4].

At ostial destinations, DES seem to have proportionate security to BMS. DES might be favored at area basic destinations, for example, the ostial LAD or LCx. Worry over future failure to reconnect a vessel with a jutting aortoostial stent may likewise incite choice of DES to limit requirement for re-mediation [5].

We intended to decide the highlights, indicators and the executives of ostial coronary conduit sicknesses and its administration.

\section{Material and methods \\ 2.1Patient population}

This investigation was directed on an advantageous example of 2000 patients conceded at cardiology division of
Benha University Hospitals and Tanta University Hospitals with ischemic chest torment experienced analytic catheterization, just 200 coordinated our objective populace and determined to have coronary ostial injury and experienced PCI.

The examination convention was officially inspected and endorsed by the morals council for human exploration at Benha Faculty of Medicine with educated assent acquired from all members before initiation of the investigation after exhaustive clarification of the investigation goals.

\subsection{Inclusion criteria}

Adult male or female $>18$ years' old who had been enrolled to have angina or ischemic chest pain and underwent diagnostic catheterization that showed ostial lesion whether, aorto-ostial lesions, non aorto-ostial lesions or branch ostial lesions.

\subsection{Exclusion criteria}

Patients with coronary spasm, heavy calcifications, presence of significant plaque in the ostium and patients with terminal co-morbidities such as end stage malignancy, end stage renal or liver diseases were excluded from our study.

After exclusion of non-responders, drop out participants and those with exclusion criteria, 200 subjects completed the study [this number was considered suitable enough sample for statistical analysis with significant results and correlations].

\subsection{Methods}

All patients were exposed to point by point history, including $\mathrm{CAD}$ chance variables, physical assessment, Electrocardiography [ECG] to recognize ST section and T wave variations from the norm.

Blood tests were gathered before heart catheterization. Patients fasted for $>12$ hours before cardiovascular catheterization. Blood was gathered either from the antecubital vein or inhabiting catheter into Two $3.2 \%$ 
trisodium citrate tube subsequent to disposing of the underlying $3 \mathrm{ml}$ of blood. Serum was isolated by centrifugation at $2000 \mathrm{~g}$ for 15 minutes and put away at $70^{\circ} \mathrm{C}$. Complete blood picture, serum creatinine, liver capacity test, electrolytes, heart biomarkers, arbitrary glucose and absolute lipid profile were estimated.

Ordinary Echocardiography proportion of the left ventricle geometry [LVEDD, LVESD, IVSd, PWDd and EF by $\mathrm{m}$-Mode] and divider movement variations from the norm utilizing Philips Echo machine and the outcomes were done indiscriminately by two reverberation specialists as suggested by American Society of Echocardiography.

Demonstrative coronary angiography was performed utilizing the percutaneous femoral methodology by Sildenger procedure. Right and left coronary angiography were performed utilizing different projections and examination was finished by proficient interventionists who were oblivious in regards to the cases.

PCI was done to ostial sores by numerous bifurcation procedures distinguishing guilty party vessel, site of sore utilizing distinctive inflatable dilatation for primary and side branches, wires, catheters and stents. At that point CA result as in regards to TIMI III stream and Residual insignificant luminal distance across [MLD] in the stent were recorded.

At last, follow up by rehashed revascularization as respects target injury revascularization [TLR] and non-target sore revascularization [Non-TLR].

\subsection{Statistical examination}

Measurable investigation was performed utilizing Statistical Package for The Social Sciences Version 22 [IBM Corp., Armonk, NY, USA]. Quantitative information are communicated as means and standard deviations.

P-Value $\leq 0.05$ was considered to show hugeness. Relationship investigation surveys the quality of relationship between two factors. Various calculated relapse investigation was utilized to identify the relationship between lipid profile and both maternal and neonatal inconveniences.

\section{Results}

According to the angiographic data, there were 54\% males while $46 \%$ of patients were females. Regarding to risk factors $58 \%$ were hypertensive, $58 \%$ were diabetics, $38 \%$ were smokers, $26 \%$ were with positive family history and $56 \%$ had dyslipidemia Table (1).

Regarding to cardiovascular History in our studied group, there were $10 \%$ with previous MI. there were $24 \%$ underwent previous PCI and no patient underwent CABG. There were $4 \%$ with a history of CVS and $18 \%$ had past history of PAD Table (2).

Regarding to the clinical presentation of our patients, $12 \%$ of patients presented with stable angina, $22 \%$ presented with unstable angina, 30\% presented with NSTEMI and $36 \%$ presented with STEMI Table (3).

Regarding to Left Ventricular Ejection Fraction [EF] in studied population the mean level of EF was $50.3 \pm 9.1 \%$ Table (4).

According to the Site of bifurcation lesion, $20 \%$ of patients were with ostial CX lesion, $26 \%$ were with ostial diagonal, $28 \%$ were with ostial LAD, $8 \%$ were with ostial LM, $12 \%$ were with ostial $\mathrm{OM}$ and $6 \%$ were with ostial RCA lesion. Table (5).

Regarding to the type of guiding catheter used in PCI, Amplatz 2 guiding catheter was used in PCI of 8 patients [4\%]. EBU guiding catheter was used in PCI of 20 patients [10\%]. JL4 guiding catheter was used in PCI of 96 patients [48\%]. JR3.5 guiding catheter was used in PCI of 12 patients [6\%]. XB guiding catheter was used in PCI of 64 patients [32\%]. Table (6).

Regarding to the guiding wire used in PCI, Sion Blue guiding wire was used in PCI of 16 patients [8\%]. PT2 guiding wire was used in PCI of 60 patients [30\%]. Fielder XT guiding wire was used in PCI of 16 patients [8\%]. BMW guiding wire was used in PCI of 80 patients [40\%]. Asahi guiding wire was used in PCI of 28 patients [14\%] Table (7)

According to Pre-Dilatation used in PCI for studied patients, predilatation of the main vessel was done in 80 patients [40\%]. predilatation of the side branch was done in 108 patients [54\%] Table (8).

Regarding to Post-Dilatation used in PCI, postdilatation of the main vessel was done in $88 \%$ of patients. postdilatation of the side branch was done in $86 \%$ of patients Table (8).

Best results and less complication were associated with [Szabo] technique, while more complications were associated with [Culotte] technique with ostial lesions Table (9).

Table (1) Demographic data and risk factors among the studied groups.

\begin{tabular}{lccc}
\hline Data & & Number [200] & Ratio 100\% \\
\hline \multirow{2}{*}{ Gender } & Male & 108 & $54 \%$ \\
& Female & 92 & $46 \%$ \\
HTN & Yes & 116 & $58 \%$ \\
& No & 84 & $42 \%$ \\
DM & Yes & 116 & $58 \%$ \\
& No & 84 & $42 \%$ \\
Smoking & Yes & 76 & $38 \%$ \\
& No & 124 & $62 \%$ \\
Family History & Yes & 52 & $26 \%$ \\
& No & 148 & $74 \%$ \\
Dyslipidemia & Yes & 112 & $56 \%$ \\
\hline
\end{tabular}


Table (2) Cardiovascular History in studied population.

\begin{tabular}{lccc}
\hline Data & & Number [200] & Ratio 100\% \\
\hline \multirow{2}{*}{ Previous MI } & Yes & 20 & $10 \%$ \\
& No & 180 & $90 \%$ \\
Previous PCI & Yes & 24 & $12 \%$ \\
& No & 176 & $88 \%$ \\
Previous CABG & Yes & 0 & $0 \%$ \\
& No & 200 & $100 \%$ \\
CVD & Yes & 8 & $4 \%$ \\
& No & 192 & $96 \%$ \\
PAD & Yes & 36 & $18 \%$ \\
\hline
\end{tabular}

Table (3) Clinical presentation in studied population.

\begin{tabular}{lcc}
\hline Data & Number [200] & Ratio 100\% \\
\hline Stable angina & 24 & $12 \%$ \\
UA & 44 & $22 \%$ \\
NSTEMI & 60 & $30 \%$ \\
STEMI & 72 & $36 \%$ \\
\hline
\end{tabular}

Table (4) Lvef in studied populations.

\begin{tabular}{lcc}
\hline Data & Range & Mean \pm SD \\
\hline EF \% & $32-68$ & $50.3 \pm 9.1$ \\
LVEDD & $4.6-5.6$ & $0.72 \pm 5.13$ \\
LVESD & $2.7-4.3$ & $0.76 \pm 3.87$ \\
\hline
\end{tabular}

Table (5) Site of Bifurcational lesion in studied population.

\begin{tabular}{lcc}
\hline Bifurcational lesion & Number [200] & Ratio 100\% \\
\hline Ostial CX & 40 & $20 \%$ \\
Ostial Diag & 52 & $26 \%$ \\
Ostial LAD & 56 & $28 \%$ \\
Ostial LM & 16 & $8 \%$ \\
Ostial OM & \multicolumn{2}{c}{$12 \%$} \\
Ostial RCA & 24 & \multicolumn{2}{c}{$6 \%$} \\
\hline
\end{tabular}

Table (6) Type of Guiding Catheter used in PCI for studied population.

\begin{tabular}{lcc}
\hline Guiding Catheter & Number [200] & Ratio 100\% \\
\hline Amplatz 2 & 8 & $4 \%$ \\
EBU & 20 & $10 \%$ \\
JL4 & 96 & $48 \%$ \\
JR3.5 & 12 & $6 \%$ \\
XB & 64 & $32 \%$ \\
\hline
\end{tabular}

Table (7) Type of Guiding Wires used in PCI for studied population.

\begin{tabular}{lll}
\hline Guiding Wires & Number [200] & Ratio 100\% \\
\hline Sion Blue & 16 & $8 \%$ \\
PT2 & 60 & $30 \%$ \\
Fielder XT & 16 & $8 \%$ \\
BMW & 80 & $40 \%$ \\
Asahi & 28 & $14 \%$ \\
\hline
\end{tabular}


Table (8) balloon dilatation among the studied population.

\begin{tabular}{lcc}
\hline Pre - Dilatation & Number [200] & Ratio 100\% \\
\hline Main Vs & 80 & $40 \%$ \\
Side Branch & 108 & $54 \%$ \\
Post - Dilatation & & \\
Main Vs & 176 & $88 \%$ \\
Side Branch & 172 & $86 \%$ \\
\hline
\end{tabular}

Table (9) Clinical outcomes of the studied populations with different techniques.

\begin{tabular}{lccccccc}
\hline $\begin{array}{l}\text { Clinical } \\
\text { Outcome }\end{array}$ & Szabo & TAP & $\begin{array}{c}\text { DK } \\
\text { crush }\end{array}$ & Mini crush & Culotte & V stenting & P value \\
\hline Arrhythmias & 5 & 12 & 14 & 5 & 11 & 8 & \\
Reinfarction & 0 & 2 & 5 & 3 & 4 & 2 & \\
HF & 4 & 7 & 6 & 9 & 8 & 9 & 0.01 \\
Death & 0 & 0 & 0 & 0 & 0 & 0 & \\
Stroke & 1 & 3 & 2 & 0 & 1 & 1 & \\
\hline
\end{tabular}

\section{Discussion}

Coronary bifurcation accounts for $15 \%$ to $20 \%$ of all percutaneous coronary intercessions [PCIs] and stays among the most testing sores in interventional cardiology regarding procedural accomplishment just as long haul clinical results [6] .

Coronary vein bifurcation sores contain one of the more mind boggling injury subsets routinely looked in interventional cardiology, representing up to $20 \%$ of all coronary infection rewarded by percutaneous coronary mediation [7].

The treatment of stenosis at an ostial sore stays one of the most testing injury subsets in coronary angioplasty. Bifurcation sores convey a danger of side branch impediment. On account of plaque redistribution or supposed "plaque move" over the carina of the bifurcation [8].

Our investigation assessed 2000 back to back patients who had angina or ischemic chest torment alluded to the cardiology division of Benha college emergency clinics and Tanta college Hospitals with ischemic chest torment experienced demonstrative catheterization, just 200 of them had ostial injuries and finished the examination with us.

In our examination as with respect to sex dissemination, $54 \%$ were guys and $46 \%$ were females. $58 \%$ of patients were hypertensive, $58 \%$ were diabetic, $56 \%$ had dyslipidemia and $38 \%$ were smokers.

Routledge et al expressed in their examination that 188 patients had dyslipidemia [76\%], 142 patients had hypertension [57\%] and 42 patients were current smokers [17\%], 31 patients had diabetes [13\%] [9].

Hypertension was a typical hazard factor in our investigation as it was a hazard factor in $58 \%$ of our patients which is concordant with spoken to $57 \%$ and $53 \%$ in Routledge et al and the Nordic examinations separately. [9, 10]

Diabetes was increasingly regular among the patients tried out our investigation than the patients selected Routledge et al. It spoke to a hazard factor in $58 \%$ of our patients while it just spoke to $13 \%$ of the patients in
Routledge et al, this distinction might be because of diabetes influences roughly $9.3 \%$ of the Egyptian populace and Egypt is required to have the biggest number of individuals with diabetes constantly 2025 [11].

As with respect to smoking, it was high among the patients joined up with our examination than the patients tried out Routledge et al and the Nordic investigations [9, 10]. This might be because of high commonness of smoking among Egyptian individuals.

As to cardiovascular history in our considered gathering, there were 20 patients [10\%] with past MI, 24 patients [24\%] experienced past PCI and no patient experienced CABG. There were 8 patients [4\%] with a background marked by CVS and 36 patients with a level of $18 \%$ had previous history of PAD.

Contrasted with our investigation, Adriaenssens et al. among his investigation populace found that $28 \%$ with earlier history of MI and $6.1 \%$ with earlier history of CABG in his contemplated populace [12].

In our investigation as in regards to Left Ventricular Ejection Fraction $[\mathrm{EF}]$ in examined populace the mean degree of EF was $50.3 \pm 9.1 \%$.

In an examination by Hildick-Smith et al, 96.4\% of patients had great left ventricular capacity [EF>50\%], 3.6\% of patients had moderate left ventricular capacity [EF30$50 \%$ ] and no patients had helpless left ventricular capacity [EF<30\%] [13].

As per the Site of bifurcation sore, $20 \%$ of patients were with ostial CX injury, 26\% were with ostial diag, $28 \%$ were with ostial LAD, $8 \%$ were with ostial LM, $12 \%$ were with ostial OM and 6\% were with ostial RCA sore.

Burzotta et al. expressed that, $49.5 \%$ of patients with LAD/D, $10.9 \%$ of patients with LCX/OM, $2.7 \%$ of patients with RCA/PDA and $36.8 \%$ of patients with left fundamental bifurcation [14].

As indicated by Pre-Dilatation utilized in PCI in our investigation, predilatation of the principle vessel was done in $40 \%$ of patients. predilatation of the side branch was done in $54 \%$ of patients. 
While as with respect to postdilatation utilized in PCI, postdilatation of the fundamental vessel was done $88 \%$ of patients. postdilatation of the side branch was done in $86 \%$ of patients. last kissing inflatable was effectively done in $80 \%$ of patients.

In the CACTUS [coronary bifurcations: utilization of the devastating method utilizing Sirolimus eluting stents] study, in the temporary stenting gathering, expand pre dilatation of the fundamental branch was done in $90.8 \%$ of patients while swell pre dilatation of the side branch was done in $90.8 \%$ of patients [15].

Our outcome was concordant to Siotia et al, who indicated that concurrent kissing stents to treat unprotected left fundamental bifurcation illness had $99 \%$ procedural achievement and gives great outcomes in commonplace back to back populace of patients with bifurcation LMS infection and furthermore might be because of spending estimating with bigger inflatables in 16\% [16] .

Sharma et al. inferred that the concurrent kissing stents method was related with more noteworthy procedural accomplishment of the side branch, with less post procedural lingering stenosis and with less technique time, which was ascribed to the set number of patients [17].

Our outcome was concordant with Burzotta et al. that last kissing inflatable expansion is fitting to reshape the situation of the jutting SB stent swaggers to make a solitary stent's swagger, little, neocarina, not influencing the MV lumen [14] .

Our outcomes additionally demonstrated that there was likewise a huge distinction between factor Ostial sores PCI Techniques with respect to Coronary Angiographic information. Best Coronary Angiographic information and less intricacy were related with [Szabo] procedure, while more complexities were related with [Culotte] strategy.

This outcome is conflicting with Lianglong et al, who found the quick achievement angiographically [residual stenosis $<20 \%$ with grade III TIMI flow] was accomplished in all patients with $100 \%$ fruitful last kissing inflatable and changed Culotte strategy is appropriate for all edges of bifurcations and gives close to consummate inclusion of the SB ostium at the site of bifurcation which is like our outcomes [18].

While Chen et al, as of late found that both smash and culotte strategy in rewarding coronary bifurcation injury had method achievement rate $96.7 \%$ and $98.7 \%$ separately [19]

Chen et al, who found following 8-month development, TLR was found in 11 patients of the twofold kissing pound bunch when contrasted with 22 patients in the great smash bunch [20.6\% versus $10.5 \%, \mathrm{P}=0.043]$. A critical distinction in TLR was kept up at a 2-year follow-up [23.4\% versus $11.4 \%, \mathrm{P}=0.022]$ with lower aggregate MACE in the kissing pound bunch contrasted with the exemplary smash bunch [29.9\% versus $18.1 \%, \mathrm{P}=0.044]$ [19] .

Hildick-Smith et al, established that there was post method stenosis in the side branch in 37 patients [33\%] [13]

In the Nordic I bifurcation study, at a half year development, in the arm of straightforward technique there were 2 instances of cardiovascular demise [1\%],4 instances of target vessel revascularization [1.9\%],4 instances of target injury revascularization [1.9\%], an instance of stent apoplexy $[0.5 \%]$ while there were no instances of myocardial dead tissue [20].

\section{Limitations}

Our investigation had a few impediments, its review nature, and absence of randomization with respect to stenting methodology left to the administrator's circumspection. Albeit randomized preliminaries are hard to complete in light of the fact that every patient's life structures may support a specific strategy, yet preliminary patients in general will be heterogeneous and furthermore the meaning of coronary conduits ailment depended on angiographic sees y 2D X-beam, we didn't utilize IVUS or FFR which may meddle with the choice of the translation of coronary angiography.

\section{Conclusion}

[Szabo] technique in ostial lesions had the best results and less complication, while more complications were associated with [Culotte] technique. Routine use of final kissing balloon did not improve clinical outcome, but there was not a penalty for undertaking final kissing balloon in patients with bifurcation lesions managed with single stent strategy.

\section{References}

[1] P. Jokhi, N. Curzen, Percutaneous coronary intervention of ostial lesions. EuroIntervention, Vol.5(4), PP. 511$514,2009$.

[2] A. Alame, E. Brilakis, Best practices for treating coronary ostial lesions. Catheterization and Cardiovascular Interventions, Vol.87(2), PP. 241242,2016.

[3] S.W. Yang, Y. J. Zhou, Percutaneous coronay intervention for ostial occlussion lesion with an anomalous right coronary artery. J Geriat Cardiol, 2009, 6: 189-193.

[4] T. W. Kwan, D. James, Y. Huang, Perfection of precise ostial stent placement. Journal of Invasive Cardiology, Vol.24.7.PP. 354,2012.

[5] P. Capranzano, A. Sanfilippo, F. Tagliareni, Long-term outcomes after drug-eluting stent for the treatment of ostial left anterior descending coronary artery lesions. American heart journal,Vol.160(5), PP. 973-978,2010.

[6] U.Retsky, F. Barry, Apparatus and method for treatment of bifurcation lesions. U.S. Patent No, Vol.8,PP.900,207, 2014.

[7] Michael Magro, C. Girasis, A.L. Bartorelli, Acute procedural and six-month clinical outcome in patients treated with a dedicated bifurcation stent for left main stem disease: the TRYTON LM multicentre registry. EuroIntervention, Vol.8(11), PP. 1259-1269,2013.

[8] P. S. Song, Y. B. Song, G. H. Yang, Periprocedural myocardial infarction is not associated with an increased risk of long-term cardiac mortality after coronary bifurcation stenting. International journal of cardiology,Vol. 167(4), PP. 1251-1256,2013. 
[9] H.C. Routledge, M.C. Morice, T. Lefevre, 2-year outcome of patients treated for bifurcation coronary disease with provisional side branch $\mathrm{T}$-stenting using drug-eluting stents. JACC: Cardiovascular Interventions, Vol.1(4), PP. 358-365,2008.

[10] S. Silber, The Nordic bifurcation study. Randomized study on the question of Birfurcation stenosis stent technique, Vol.30[15] , PP.113-125,2006.

[11] M. Bos, C. Agyemang, Prevalence and complications of diabetes mellitus in Northern Africa, a systematic review. BMC public health, Vol.13(1), PP.387,2013.

[12]T. Adriaenssens, R. Byrne, A. Dibra, Culotte stenting technique in coronary bifurcation disease: angiographic follow-up using dedicated quantitative coronary angiographic analysis and 12-month clinical outcomes. European heart journal, Vol.29(23), PP. 28682876,2008

[13]D. Hildick-Smith, A. DE Belder, N.Cooter, Randomized trial of simple versus complex drug-eluting stenting for bifurcation lesions, Vol. 25(8), PP.121,2010.

[14]F. Burzotta, H. C. Gown, H. Y. Hahn, Modified Tstenting with intentional protrusion of the side-branch stent within the main vessel stent to ensure ostial coverage and facilitate final kissing balloon: The $\mathrm{T}$ stenting and small protrusion technique [TAP-stenting]. Report of bench testing and first clinical Italian-Korean two-centre experience. Catheterization and
Cardiovascular Interventions, Vol.70(1), PP. 7582,2007.

[15]A. Colombo, E. Bramucci, S. Sacca, CLINICAL PERSPECTIVE. Circulation, Vol. 119(1), PP.71$78,2009$.

[16] A. Siotia, A. C. Morton, C. Malkin, Simultaneous kissing drug-eluting stents to treat unprotected left main stem bifurcation disease: medium term outcome in 150 consecutive patients. EuroIntervention,Vol.8(6), PP. 691-700,2012.

[17] S. K. Sharma, A. Choudhury, J. Lee, Simultaneous kissing stents [SKS] technique for treating bifurcation lesions in medium-to-large size coronary arteries. The American journal of cardiology, Vol.94(7), PP.913917,2004.

[18] S.C. Liang, X.u. Bo, Han Yaling, Comparison of double kissing crush vs culotte stentingfor distal left main JACC, Vol.24, PP. 124-138, 2013.

[19] Shao-Liang Chen, B. Xu, I. Sheiban, Clinical outcome after DK crush versus culotte stenting of distal left main bifurcation lesions: the 3-year follow-up results of the DKCRUSH-III study. JACC: Cardiovascular Interventions, Vol.8(10), PP. 1335-1342, 2015.

[20]M. Niemelä, K. Kervinen, A. Erglis, Randomized comparison of final kissing balloon dilatation versus no final kissing balloon dilatation in patients with coronary bifurcation lesions treated with main vessel stenting: the Nordic-Baltic Bifurcation Study III. Circulation, Vol.123(1), PP.79-86,2011. 